\title{
Interoperability Framework for Supporting Information-Based Assistance in the Factory
}

\author{
Mohamed Anis Dhuieb, Farouk Belkadi, Florent Laroche, and Alain Bernard \\ L'UNAM Université, IRCCYN, Ecole Centrale de Nantes, \\ 1 rue de la Noë, 44300 Nantes, France \\ mohamed-anis.dhuieb@irccyn.ec-nantes.fr
}

\begin{abstract}
The aim of this paper is to propose new interoperability solution, based on Info-Engine framework and web services technology to support data exchange and extraction from PLM system, specially the Windchill tool. This solution will be implemented as a connector module of more generic framework, named Digital Factory Assistant (DFA). The DFA framework aims to provide factory workers by a set of knowledge and information based decision support to improve their activity performance.
\end{abstract}

Keywords: Product Lifecycle Management, Interoperability, Knowledge and Data extraction, Windchill.

\section{Introduction}

Visions about the factory of the future affirm that future production lines will be characterized by learning capability and decisions support in all stages of the process chains involved. The aim of such propositions is to achieve higher manufacturing outputs by integrating knowledge-based modules into the engineering systems concerned in the factory. Despite the evolution of digital tools in the factory, the role of the human being in the whole product lifecycle development remains a key factor to be continuously improved by means of several actions of training and assistance. For this, one of the main challenges in today's production systems is to provide factory actors with robust tools helping them to perform more efficiently their daily activities and to enhance their reactivity in front of critical working situations.

The proposal of this paper is developed as a part of the ARTUR project that aims to deal with the topic of worker assistance through the development of the "Digital Factory Assistant" (DFA). By means of this assistant, the operator will benefit from an omnipresent support along the manufacturing process. The DFA restitutes knowledge from distributed sources and relies on different ways of interaction with the user in order to provide him relevant information and decision supports.

Different knowledge sources can be considered in the factory, such as Product Lifecycle management system (PLM), CAx tools (Computer aided Design/ Manufacturing/engineering CAD/CAE/CAM...), material flows, process chains, and simulation data. The basic idea of the DFA concept is to allow each person being able 
to respond on any given situation, mainly thanks to high-speed simulation and performance evaluation models and methods. For that need, an interoperability framework is proposed, aiming to support information extraction and knowledge routing in the factory.

The next section of the paper describes the idea behind the ARTUR project and the proposed Digital Factory Assistant framework. In the third section, we made a state of the art about the interoperability issue within recent research works and in PLM context. The fourth section describes the proposed framework of the PLM connector and then, the last section includes some perspectives of the current work.

\section{The Concept of "Digital Factory Assistant"}

In a production system, the worker may be faced with critical working situations that require an efficient reaction by making the right decision in the right time. As it is described in the introduction, the aim of the DFA is to support the factory workers to react in front of difficult tasks by providing them the right information and knowledge using advanced methods and technologies supporting information visualization and manufacturing process simulation.

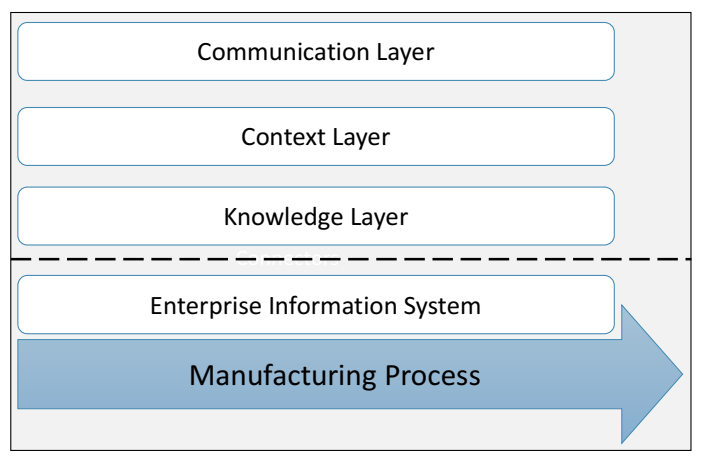

Fig. 1. Conceptual architecture of the Digital Factory Assistant

As shown in figure 1, the generic conceptual architecture of the DFA is composed of three main layers:

- Communication layer: the communication layer deals with the human machine interactions and the problem of information and knowledge representation. Virtual technologies are used to propose a better restitution of the information. This layer implements also advanced simulation interfaces connected to the manufacturing process simulation module, to guarantee a better understanding and control of the process. Thanks to this simulation interface, the user can manipulate some parameters of the manufacturing process and observe the outcome of the simulated process before committing on the real working situation. 
- Context layer: the context layer is an integration of the working situation [1]. This layer is related to the human-machine level, where contextual information related to user's working situation can be acquired. The proposal of this assistant is based on context awareness issue [2]. Context-aware systems have the particularity of anticipating the user's needs in a particular situation and act proactively to provide appropriate assistance [3]. Contextual information can be acquired from real working situation and part of it may be extracted from the manufacturing enterprise information system.

- Knowledge layer: The role of this layer is to introduce a new way of definition, representation, and exploration of knowledge in the factory. The main idea is to provide worker by useful knowledge according to a multilevel structure, in which each level represents the completeness degree of knowledge [4]. The workers performance will be measured before and after using the DFA in daily activities and in front of difficult tasks in order to evaluate their improvement using the DFA.

Regarding the functional architecture of the DFA (figure 2), several interaction modes are preconized to provide data and knowledge capitalization and reuse. The first category of interactions concerns the communication between the user and the treatment module of the digital assistant throughout augmented reality and virtual reality interfaces. According to this interaction, user input his request and the DFA provide user useful assistance regarding his request, his profile and his working situation. The second category of interaction concerns data exchange between the DFA and other existing enterprise information systems through specific connectors. The third category of interactions, between the treatment module, solution finding and knowledge base, aims to perform knowledge reuse purposes. The last category supports the purpose of knowledge capitalization from both experts and external simulation software.

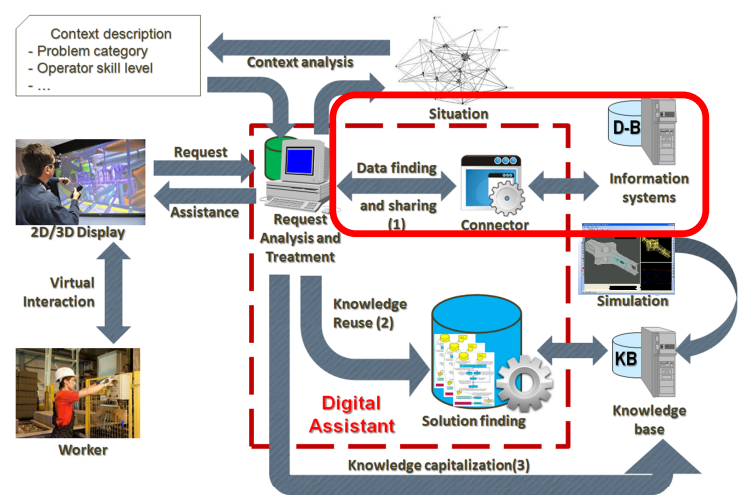

Fig. 2. Functional architecture of the digital factory assistant

To perform data exchange and extraction actions, interoperability mechanisms are required as fundamental capacities of the connector module, for each kind of 
information system to be connected. This paper deals with the interoperability issue and focus on the definition of a technical solution in order to support interoperability with PLM System and particularly, the Windchill PLM solution.

Before presenting the detailed architecture of the interoperability solution, the following section presents an overview of related works in the field of interoperability in PLM context.

\section{Interoperability with PLM Systems: A State of the Art}

Generally, three scopes of interoperability are distinguished with different terms and definitions. For instance: 1) the Conceptual interoperability that concerns the definition of concepts and semantic supporting the communication between data and knowledge models; 2) the Organizational interoperability, which focuses on the connection between processes and 3) the technical interoperability that deals with technological issues to support data exchange between software applications.

In PLM domain, three major approaches are currently used to support the interoperability and data exchange between Computer Aided applications (CAX) (X such as Design, simulation, etc.) and between CAX and data management systems.

\subsection{Ontology and Web Semantic Technologies}

The first category uses the ontology and Web Semantic technologies to achieve the data mapping between heterogeneous software at the conceptual level. Several studies implementing different approaches to product design have been conducted on Ontology, as standard for data exchange between design and other engineering activities in collaborative tasks [5]. In PLM field, [6] have proposed the ONTO-PLM framework, as a common core model to provide an interoperability solution between product data (encapsulated in PLM) and enterprise applications that will manage them such as ERP, CAD and MES. The conceptual framework consists in conceptualizing of existing standards, principally were ISO 10303 and IEC 62264, related to product technical data modeling providing a "product-centric" information model to concepts that can be processed by several enterprise applications. Model driven and Knowledge based architecture is also advocate supporting the semantic interoperability between PLM systems and other applications [7].

\subsection{Standard-Based Approaches}

The second category uses a standard-based mechanism, such as XML (eXtensible Markup Language), to guarantee the semantic translation between heterogeneous models [8]. For instance, in the ATHENA project, process standards (ISO15288, CMII) and product standards (STEP: STandard for the Exchange of Product model data. Ex: AP214, 233, 209, 239) have been studied [9]. IGES (Initial Graphics Exchange Specifications) and DXF (Drawing Exchange Format) standards are also used to manage the geometric data of the product [10]. 
In PLM field, the PLCS (Product LifeCycle Support) correspond to the STEP AP239 standard realized by the International Standard Organization in 2005 [11] to offer a generic framework for the integration, exchange and management of technical data necessary for the support of a complex product and its evolution along its whole lifecycle. Based on this standard, [12] have proposed an interoperability framework to perform data mapping between ERP and PLM.

\subsection{API Standards and Web Services}

The last category uses dynamic interfaces, based on API Standards (Application Programming Interface) and web services technologies, to guarantee the communication between software [13]. In this kind of interoperability mechanism, software integration is fulfilled through the web services to support the distribution of heterogeneous information between members of a project team. In PLM field, "OMG PLM Enablers" based on middleware technologies and "PLM Services" are Web technologies developed to support communication between PLM systems and between PLM and other CAX applications [14].

The specification of the PLM Services 1.0 defines a Platform Independent Model (PIM) for Product Lifecycle Management Services. This specification defines a Platform Specific Model (PSM) applicable to a Web services implementation defined by a WSDL specification, with a SOAP binding, and an XML schema specification [15]. For instance, in [16], an XML-based neutral file is defined by referring to PLM services for the building of PLM integrator as software that can exchange product, process and resource information between commercial PLM systems. The PLM integrator consists of the XML adapter and the PLM adapter to extract PPR (Product Process Resources) information from a commercial PLM system.

PLM Services standard is used in another work by [17] to support data exchange between two different PLM systems via Internet; an implementation is fulfilled to support interoperability between two commercial PLM solutions: SmartPLM and DynaPLM. In this architecture, the product data inside the PLM system is translated to PLM Services reference server by the data transfer module. The data transfer module captures the PLM system data by using APIs and translates it into the format which PLM Services reference implementation can read and visualize. Any permitted XPDI client of PLM Services from the remote side can access the translated PLM data in the PLM Services server.

The work developed in this paper can be included in the last category of interoperability approaches. The aim is to develop a technical framework exploiting the features of the Info*Engine framework provided by PTC and the SOA standards.

\section{$4 \quad$ PLM Connector Framework}

Windchill, developed by PTC (Parametric Technology Corporation), is a PLM system offering to users a large variety of tools to support different aspects of their collaborative development projects and data and document management. Regarding 
the growing interest in interoperability, PTC has embraced Open Standards, such as Service Oriented Architecture (SOA), as its strategy for supporting integration between the PLM and other IT applications. For this need, Windchill, exposes functionality for purposes of integration through a standards-compliant Web Services framework populated with an extensive library of prebuilt services.

\subsection{Info*Engine $®$ Architecture}

Windchill integration with other enterprise's applications requires the use of low-level APIs and complex application adapters. Support new integration scenarios is a labour task and requires strong developpment skills. For this need, Windchill Info*Engine server provides mechanisms for retrieving and manipulating the data that users or custom applications want to view or receive from the PLM Server.

As shown in figure 3, the proposed architecture is based on Info*Engine Java 2 Enterprise Edition (J2EE) that is an implementation of J2EE Connector Architecture (JCA). JCA was designed to supply standard implementation for interaction between J2EE application servers and enterprise information systems (EIS).

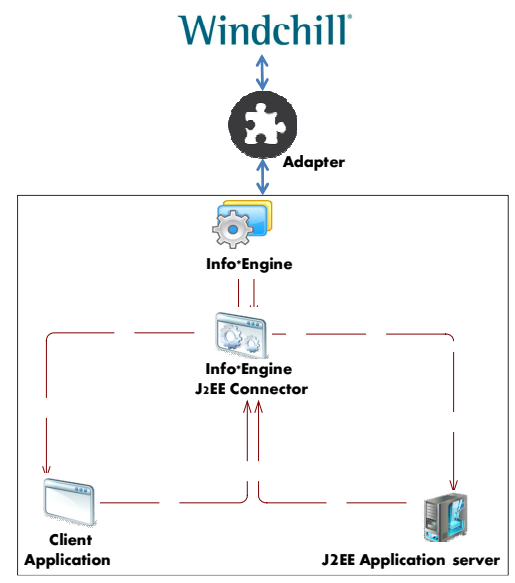

Fig. 3. The info*Engine based architecture

The Info*Engine J2EE connector uses SOAP protocol to allow communication between Info*Engine and the two modules of the interoperability application. For instance, J2EE application server and associated client application are developed as an integrated solution for the interoperability purpose.

The client application is deployed on Oracle $11 \mathrm{~g}$ application server. By means of this client application, the user can perform different interoperability actions like creating/deleting an object in the Windchill server or even adding a link between a part and a document. The J2EE application server support the interperetation and execution of actions prescripted by the client application. Otherwise, Info*Engine is directly interfaced with Windchill application by means of the Native Adapters component. The implementation of the proposed solution is achieved by a set of 
interactions between the different components of Info*Engine framework and those of the interoperability client application.

As it is shown in figure 4, the client application communicates directly with the Info*Engine SOAP Servlet that catch and process SOAP requests and send in the same protocol the required information to the client application. For this need, the SOAP servelt invokes tasks execution on the SAK (Service Access Kit), which is an API facilitating the development of Java applications using of Info*Engine functions and features.

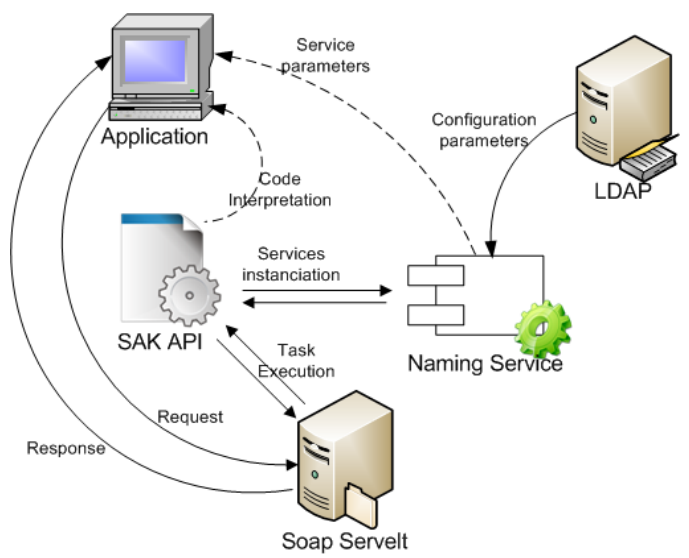

Fig. 4. Internal and external interactions in the proposed framework

During task execution, SAK interacts with the naming service in order to instantiate the required services. With the Naming Service, SAK can identify in the LDAP directory all network addresses and configuration properties. In the meantime, the client application has a direct connection to the SAK and the naming services to extract the services parameters and code interpretation respectively, which are required for the definition of the Webservice request.

\subsection{Interactions Process}

Based on the technical architecture presented above, a webservices based application is developed. Figure 5 illustrates the interactions schema between the user, the interoperability client application and Windchill, throughout the Info*Engine framwork.

After the user identification by entering his Windchill login and password, the client application receives from Windchill a list of different objects that are associated to the user role in the database.

At the right of the interface, a set of authorized actions are proposed to the user to perform on the Windchill server throughout the Info*Engine based framework. For instance, the user can obtain more details about the selected object, check out, modify or delete this object or also, creates link with other Windchill objects. 


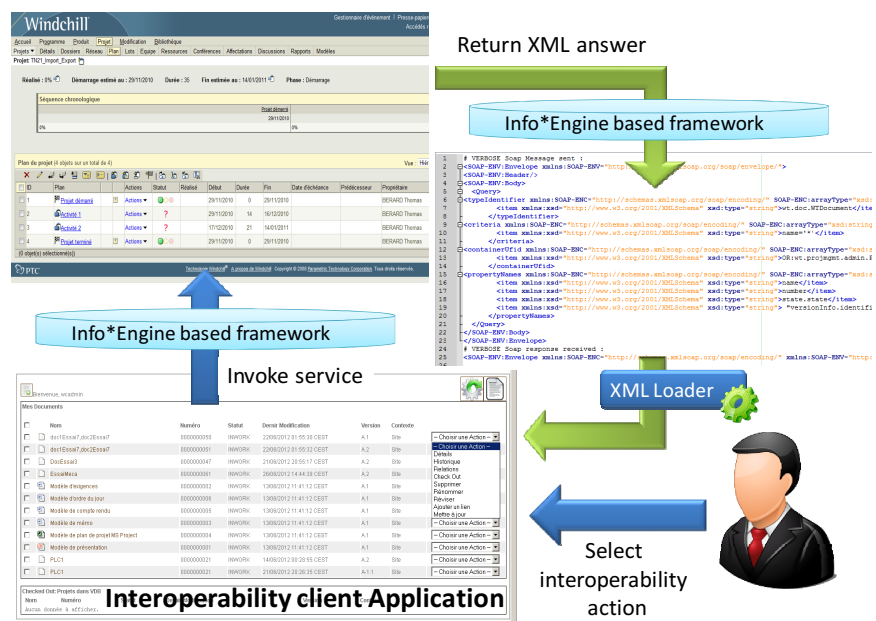

Fig. 5. Webservice-based application

When the user validates his action, the client application invoke the corresponding service(s) on the info*engine framework according to the general interaction schema presented in figure 5. Then, the Windchill send the answer in a predefined XML file to the info*Engine framework. It will be a list of information about the selected object, a validation message about the modification or deleting a specific object or also a notification of a denied action.

\section{Conclusion and Future Work}

Windchill is one of the most popular PLM software, which proposes robust solutions to support communication with external information systems. The purpose of this paper is first to prospect the interoperability mechanisms offered by PTC editor as a solution to interoperability issue.

Then, a new interoperability framework is proposed as a web-service based solution using the capacities embedded on the Windchill Info*Engine mechanisms and J2EE application technology.

The first results of the interoperability tests performed on the proposed framework illustrate a high level of reactivity to different requests. The main advantages of the proposed architecture is that it offer a large possibilities to pilot the communication with Windchill server through an external third-party application while ensuring a coherence with internal mechanisms of Windchill, since it exploits directly the internal components of Windchill.

This framework is a first step for the development of an integrated connector for Windchill server. The main utility of this connector is to handle process and product knowledge to be integrated in a virtual decision support system. Further work will aim at the definition of robust loader tool to support the interpretation of Windchill responses by the treatment module of the Digital Factory Assistant. 
Acknowledgements. This work is financed by the region of Pays de la Loire (France). The research project involves four teams from Ecole Centrale de Nantes (IS3P and PsyCoTec teams from IRCCyN laboratory, GEM, and CERMA), and one virtual Reality applications integrator CLARTE. Particular thanks are addressed to the industrial partner, for accepting the validation of this scientific proposal.

\section{References}

1. Hasan, R., Bernard, A., Ciccotelli, J., Martin, P.: Integrating safety into the design process: elements and concepts relative to the working situation. Safety Science 41(2-3), 155-179 (2003)

2. Schmidt, A.: Context-Aware Computing: Context-Awareness, Context-Aware User Interfaces, and Implicit Interaction, 2nd edn. The Encyclopedia of Human-Computer Interaction (2013)

3. Laroche, F., Bordeu, F., Bernard, A., Chinesta, F.: Towards the factory of future An integrated approach of material-processes-information-human being. In: Proceedings of the 2012 Virtual Reality International Conference, p. 13. ACM (2012)

4. Dhuieb, M.A., Laroche, F., Bernard, A.: Toward a cognitive based approach for knowledge structuring. In: 2013 IEEE 4th International Conference on Cognitive InfoCommunications (CogInfoCom), pp. 407-412. IEEE (December 2013)

5. Bellatreche, L., Xuan, D.N., Pierra, G., Dehainsala, H.: Contribution of Ontology-based Data Modeling to Automatic Integration of Electronic Catalogues within Engineering Databases. Computers in Industry 57(8-9), 711-724 (2006)

6. Panetto, H., Dassisti, M., Tursi, A.: ONTO-PLM: Product-driven ONTOlogy for Product Data Management interoperability within manufacturing process environment. Advanced Engineering Informatics 26, 334-348 (2012)

7. Bermell-Garcia, P., Fan, I.S., Murton, A.: Towards the semantic interoperability between KBE and PLM systems. In: International Conference on Engineering Design, cite des Sciences et l'industrie, Paris, France, August 28-31 (2007)

8. Chen, D., Vernadat, F.: Standards on enterprise integration and engineering: A state of the art. International Journal of Computer Integrated Manufacturing 17(3), 235-253 (2004)

9. Ruggaber, R.: ATHENA - Advanced Technologies for Interoperability of Heterogeneous Enterprise Networks and their Application. In: International Conference on Interoperability of Enterprise Software and Applications, Geneva, Switzerland, February 23-25 (2005)

10. Choi, G.H., Mun, D., Han, S.: Exchange of CAD Part Models Based on the MacroParametric Approach. International Journal of CAD/CAM 2(1), 13-21 (2002)

11. Rosen, J.: Federated through life-cycle support. In: 1st Nordic Conference on PLM, NordPLM 2006, Göteborg, Sweden, January, 25-26 (2006)

12. Paviot, T., Cheutet, V., Lamouri, S.: A PLCS framework for PLM/ERP interoperability. International Journal of Product Lifecycle Management 5(2/3/4), 295-313 (2011)

13. Song, H., Roucoules, L., Eynard, B., Lafon, P.: Interoperability between Cooperative Design Modeller and a CAD System: Software Integration versus Data Exchange. International Journal for Manufacturing Science \& Production 7(2), 139-149 (2006)

14. Wang, Y., Ge, J., Shao, J., Han, S.: Research on Web Service-based Interoperability of Heterogeneous PLM Systems. In: International Conference on Measuring Technology and Mechatronic Automation, Hunan, China, April 11-12 (2009) 
15. Lukas, U., Nowacki, S.: High Level Integration based on the PLM Services Standard. In: ProSTEP iViP Science Days 2005, September 28-29, pp. 50-61. Cross-Domain Engineering, Darmstadt (2005)

16. Choi, S.S., Yoon, T.H., Noh, S.D.: XML-based neutral file and PLM integrator for PPR information exchange between heterogeneous PLM systems. International Journal of Computer Integrated Manufacturing 23(3), 216-228 (2010)

17. Gunpinar, E., Han, S.: Interfacing heterogeneous PLM systems using the PLM Services. Advanced Engineering Informatics 22, 307-316 (2008) 